\title{
INVESTIGATION INTO THE HYDRODYNAMIC PROCESSES OF FITTING CONNECTIONS FOR DETERMINING PRESSURE LOSSES OF TRANSPORT HYDRAULIC DRIVE
}

\author{
Mykola KARPENKO*, Marijonas BOGDEVIČIUS \\ Dept of Mobile Machinery and Railway Transport, Vilnius Gediminas Technical University, Lithuania
}

Received 16 December 2019; revised 5 February 2020, 2 March 2020; accepted 20 March 2020

\begin{abstract}
The article presents the findings of theoretical and experimental research on hydraulic processes occurring in the hydraulic drives of transport machines. The paper analyses the influence of hydrodynamic processes on the flow characteristics of fluid considering different hydraulic fitting connections. The performed analysis is based on numerical simulations using Navier-Stokes equations for the velocity field. The dynamics of fluid flow in the hydraulic system has been investigated taking into account the main parameters like system flow rate in the range of 5 to $100 \mathrm{~L} / \mathrm{min}$, the diameter of the pipeline making 1/2" and fitting standards DKOL, ORFS, BSP and JIS. As a result, pressure drop, power losses, resistance and flow coefficients at different fitting connections have been obtained. The article compares the provided results with the findings given employing the calculation method for the standard of equivalent length fitting. To simulate fluid flow, a mesh independence study and turbulence calculations have been performed. Simulation results have been examined conducting physical experiments on measuring pressure losses. Each experimental research includes three measurements of connections bearing in mind each fitting standard.
\end{abstract}

Keywords: pipeline, fittings, hydrodynamics, flow coefficient, minor losses, computational fluid dynamics, fluid pressure, energy consuming, pressure losses, resistance coefficient.
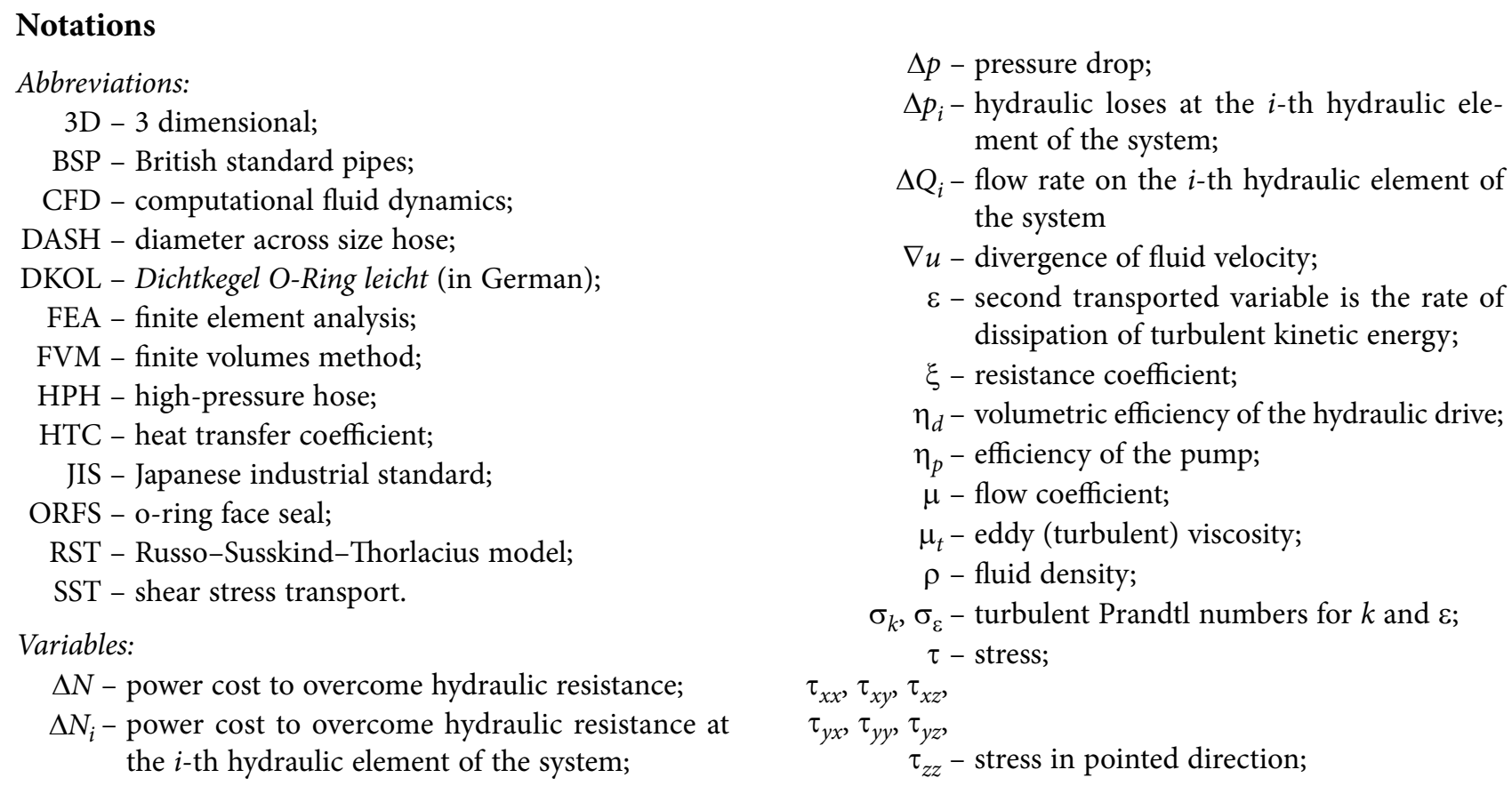

${ }^{*}$ Corresponding author. E-mail: mykola.karpenko@vgtu.lt 
$A$ - average cross-sectional area;

$C_{\mu}, C_{1 \varepsilon}, C_{2 \varepsilon}-$ constants of the $k-\varepsilon$ turbulence model;

$D$ - pipeline diameter;

$d$ - average diameter of fittings;

$E_{i j}$ - component of strain rate;

$I$ - identity matrix (unit matrix);

$k$ - first transported variable is the turbulent kinetic energy;

$m$ - number of elements in the system;

$n$ - number of fluid flow lines streams;

$N_{L}$ - power losses;

$p$ - hydraulic pressure;

$p_{0}$ - initial hydraulic pressure;

$Q$ - flow rate;

Re - Reynolds number;

$t$ - time;

$u, v, w$ - velocity components;

$u_{0}$ - velocity vector normal to the boundary;

$u_{i}$ - velocity component of the corresponding direction;

$v_{\text {average }}$ - the average velocity of fluid flow in fitting connection;

$V_{\text {in }}$ - fluid velocity at the inlet of fitting connections;

$x, y, z$ - coordinates;

$x_{i}, x_{j}$ - coordinates with index location.

\section{Introduction}

A hydraulic system of any modern heavy machinery includes a variety of different assemblies and is considered and simulated as a complex dynamic system while applying multi-criteria methods. A variety of specific elements forming the structure of each hydraulic system allows connecting hydraulic equipment, HPHs and other parts into a single system, i.e. fittings. HPHs and fittings make a connection and have the purpose of ensuring the correct direction of fluid flow inside the system (Nishimura, Matsunaga 2000). The present improvements to the hydraulic drive have been critical and thus have pressured the manufacturers to analyse carefully all factors contributing to the development of efficient hydraulic drives. Due to the stringent policy of power consumption, researchers have shifted focus on the increasing effect of the subsystems taking control over the energy consumption process, and consequently, the formation of energy saving.

Yan et al. (2019) pointed out that even low resistance in the hydraulic drive brought major influence on power consumption. Along with stricter control, a reduction in energy consumption and the optimization of hydraulic drive performance and fluid flow inside the drive have deemed as one of the major contributing factors. According to De Moraes et al. (2017), an efficient system of fluid flow in hydraulic drives in particular is a critical factor in obtaining optimum processes having an effect on power consumption.
$\mathrm{HPH}$ and fittings act as two connected objects failing to exist in the hydraulic system one without the other and are presented in Figure 1a (Karpenko, Bogdevičius 2018). Losses caused by fluid friction and sudden changes in the diameter of fittings occur in this type of the system and affect pressure and power losses in the hydraulic system. At the same time, the theoretical studies of the pressure losses of fittings normally do not consider their values that are approximate and were established experimentally in the 1980s by Crane Co (1982). The accuracy of hydraulic calculations is critical to the proper design, operation and cost of many types of hydraulic systems in mobile machines. One of the aspects leading to mistakes is the misuse of coefficients characterizing the flow characteristics of devices.

The modern hydraulic systems of transport vehicles shown in Figure $1 \mathrm{~b}$ are complex and branching (Karpenko, Bogdevičius 2018). The number of connecting fittings and HPHs used in the hydraulic drives of transport vehicles usually reaches more than one or two hundred. For example, manipulator Hangcha CPCD 160-GX35 (http://www.hcforklift.com, manufacturing was discontinued since 2015) contains $178 \mathrm{HPHs}$ with fitting connections.

The more diverse adapters and connections are used in the hydraulic system, the greater are pressure losses of the system, which negatively affects energy parameters for transport vehicle drives. The wrong selection of connections or adapters has an adverse influence on pressure losses. Also, the connections or adapters of the same type but different in diameters reduce pressure in the system in general, since more fluid is required for passing their cross-section plane (Bojko, Kozubková 2018). A significant problem is to find an effective research methodology that allow analysing influence on pressure losses and determining the resistance coefficients of real fitting connections and their effect on energy consumption of hydraulic drives.

a)

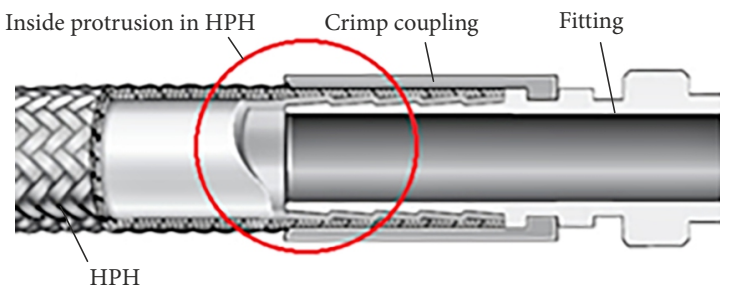

b)

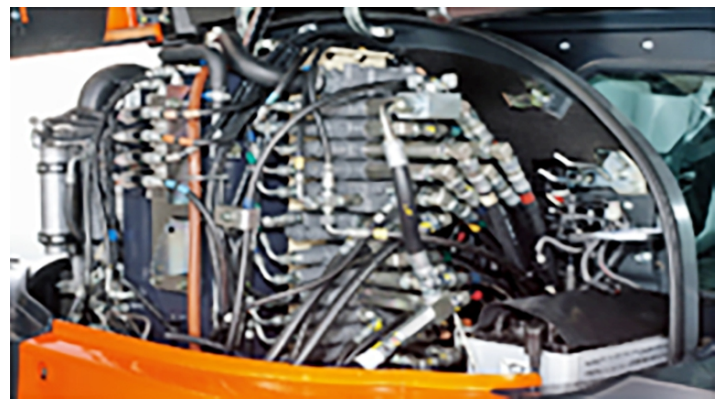

Figure 1 . The typical hydraulic composition of a system (Karpenko, Bogdevičius 2018): a - HPH with fittings; $b$ - part of hydraulics 


\section{A review of research on hydraulic fitting connections}

The analysis of fluid flow in hydraulic systems indicates some parameters used for quantifying the efficiency of elements. The most frequently used parameters include pressure drop, resistance coefficient and flow coefficient (Valdés et al. 2014). Due to changes in the size of the cross-section area, the formation of a vortex occurs at fitting connections. The main vortex generates other smaller whirlwinds carried away by the flow and at the same time disintegrates into even smaller whirlwinds. Thus, energy losses occur in the main vortex and along the length of the next section of the flow. As a result, local losses are significantly higher in the relatively short pipeline systems. Different types of research methods are used in the approach of investigating pressure drop in hydraulic systems. According to De Moraes et al. (2017), a number of methods estimate pressure drop, for example, the method of equivalent lengths, the $K$ or new crane method and the $2 K s$ method. These techniques are based on the premise that pressure drop resulting from each of the fittings is constant depending only on the diameter of that section without taking into account changes in fluid flow. De Moraes et al. (2017) explained that for calculating losses at hydraulic connections, the equivalent fitting length method could be used thus adding the length of fittings only to the length of the pipeline. The main disadvantage of all these methods is that they hardly take into account some of liquid characteristics such as viscosity, thermal analysis and compression. As for this method, we lack information whether it has had a vortex of fluid inside the HPH-fitting system and turbulent kinematic energy.

The evaluation of the characteristic of the fittings using the same describing like as valves characteristic what includes and the respective loss factors resistance coefficient and flow coefficient by Bojko and Kozubková (2018). The flow coefficient of a device is a relative measure of its ef- ficiency at the allowed fluid flow. The coefficient describes the relationship between pressure drop across an orifice, valve or other assembly and the corresponding flow rate.

According to the flow bench test done by Catellani et al. (2016), this is the most common method for determining pressure drop and the flow coefficient in the elements of the hydraulic system. The introduced technique, by fact, provides relatively accurate data on flow condition, including the flow coefficient and is used for measuring swirl and pressure drop. The main disadvantages cover its experimental investigation into the ongoing theoretical research together with CFD only. According to Li et al. (2019), the use of CFD has the advantages of simulating the investigated fluid flow in the elements of the hydraulic system.

According to Gai et al. (2019), the numerical approaches based on time step FEA and CFD methods are accepted for complicated cases. However, in 3D simulation, model setup and computations are time-consuming. CFD based on finite volume technology is used for simulating the 3D turbulent flow with a high degree of accuracy. Various turbulent models are employed for investigation purposes. A review of the above introduced models is presented in Table 1.

According to Akin and Kahveci (2019), the closest result obtained from simulating different CFD turbulent models for pressure drop to experimental research gated from standard $k-\varepsilon$ model. Taking into account time cost and resource simulation, the standard $k-\varepsilon$ model is accepted for investigating a full turbulence flow rate through fitting connections.

To overcome hydraulic resistance, engine power was expended and calculated with reference to the equation provided by Liu et al. (2020):

$$
\Delta N=\sum_{i=1}^{m} \Delta N_{i}=\frac{1}{\eta_{p} \cdot \eta_{d}} \cdot \sum_{i=1}^{m} \Delta Q_{i} \cdot \Delta P_{i} .
$$

Table 1. Review of turbulence models

\begin{tabular}{|l|l|l|l|}
\hline \multicolumn{1}{|c|}{ Model } & \multicolumn{1}{|c|}{ Reference } & \multicolumn{1}{|c|}{ Problem } & \multicolumn{1}{c|}{ Description } \\
\hline $\begin{array}{l}\text { Standard } \\
k-\varepsilon\end{array}$ & $\begin{array}{l}\text { Pechánek, } \\
\text { Bouzek (2012) }\end{array}$ & $\begin{array}{l}\text { Calculation of flow velocity, } \\
\text { pressure drop and HTC in the } \\
\text { water jacket cooling channel }\end{array}$ & $\begin{array}{l}\text { Standard of the industry and science model. Valid for fully } \\
\text { turbulent flows and any Reynolds number. Good at being used for } \\
\text { investigation on fluid flow in pipeline systems }\end{array}$ \\
\hline $\begin{array}{l}\text { Realizable } \\
k-\varepsilon\end{array}$ & $\begin{array}{l}\text { San Andres } \\
\text { et al. }(2014) .\end{array}$ & $\begin{array}{l}\text { Design of the hydrodynamic } \\
\text { process in the cooling system } \\
\text { using CFD }\end{array}$ & $\begin{array}{l}\text { Performance generally exceeds the standard } k-\varepsilon \text { model. Ineffective } \\
\text { for near wall-bounded boundary and high Reynolds number flows }\end{array}$ \\
\hline $\begin{array}{l}\text { Standard } \\
k-\omega\end{array}$ & $\begin{array}{l}\text { Khalizadeh } \\
\text { et al. }(2019)\end{array}$ & $\begin{array}{l}\text { Discovering a different effect of } \\
\text { turbulence simulating schemes } \\
\text { for wind-driven }\end{array}$ & $\begin{array}{l}k \text { - } \omega \text { models improve the performance of boundary layers as } \\
\text { compared to } k-\varepsilon \text { models. Better suitable for near wall-bounded and } \\
\text { low Reynolds number (i.e. transitional) flows }\end{array}$ \\
\hline SST $k-\omega$ & $\begin{array}{l}\text { Savkiv } \\
\text { et al. }(2019)\end{array}$ & $\begin{array}{l}\text { Optimization of design } \\
\text { parameters of Bernoulli gripper } \\
\text { with an annular nozzle }\end{array}$ & $\begin{array}{l}\text { Used for vortex flows without requiring a sublayer and predicts the } \\
\text { separation flow. Suffers from inherent limitations on the isotropic } \\
\text { eddy-viscosity model. Good at being used for aerodynamic } \\
\text { simulations determining the skin-coefficient (best at being used for } \\
\text { the wall elements of turbulence) }\end{array}$ \\
\hline RST & $\begin{array}{l}\text { Han } \\
\text { et al. }(2014)\end{array}$ & $\begin{array}{l}\text { RST model for turbulent flow } \\
\text { and heat transfer mechanism in } \\
\text { a corrugated tube }\end{array}$ & $\begin{array}{l}\text { Better predicts complex flows than eddy viscosity models, since } \\
\text { transport equations for Reynolds stresses account for the effects of } \\
\text { turbulence anisotropy and streamline curvature, but has heather } \\
\text { time cost }\end{array}$ \\
\hline
\end{tabular}


According to Liu et al. (2020) investigation into the applied equipment and the cost of five different types of the electro-hydraulic power unit, the smaller pressure drop occurs in the system, the lower is the power cost of hydraulic units.

\section{Fitting standards}

The structure of fitting connections includes two main elements: a nipple with a fixing nut and a connecting nipple. Karpenko and Bogdevičius. (2018) point out four most frequently used standards of hydraulic fitting connections: ORFS, JIS, BSP and DKOL. From the left to the right, Figure 2 presents JIS, BSP and ORFS fitting standards.

According to Karpenko and Bogdevičius (2018), for primary research in this article, the most popular standards have been selected:

- DKOL connection with a cylindrical metric thread made according to the DIN 20066:2016;

- BSP connection with a cylindrical thread made according to the BS EN 10226-2:2005;

- JIS connection with a 37-degree flare seating surface made according to the JIS B 8363:2015;

- ORFS connection with a face seal made according to the SAE J1453/3_201206 (2012).

For simulation purposes, the cross-section standards of the created 3D model fitting connections are presented in Figure 3.

The main issue is changes in the size and configuration of the cross-section area of the HPH with fitting connections (cut in the connection of HPH and DKOL fittings is shown in Figure 4).

The current research has demonstrated that $08 \mathrm{DASH}$ (inner parameter) conditional passage $\mathrm{HPH}$ will be used. Karpenko and Bogdevičius (2020) disclose that the HPH standard has a diameter of the conditional pass of $1 / 2$ " $(12.7 \mathrm{~mm})$ and is one of the most frequently used diameters of the HPH.

\section{Pre-experimental research}

The pre-experimental part of the conducted research included the analysis of pressure drop in the pipeline and fitting connections. An experimental bench for the preexperimental research is shown in Figure 5 and includes: 1 - a hydraulic tank with fluid and a hydraulic pump (inside the tank); 2 - an asynchronous motor connected to the pump; 3 - a metal pipeline for connecting the pump and the distributor valve with the safety valve; 4 - the distributor valve and the safety valve; 5 and 7 - electrical manometers with output signal; 6 - HPH; 8 - fitting connections; 9 - a throttle; 10 - a computer for data recording from manometers. Pressure in the hydraulic system is $2 \mathrm{MPa}$; flow rate is around $24 \mathrm{~L} / \mathrm{min}$; pipeline $-1 / 2$ " $(12.7 \mathrm{~mm})$; fittings connection standard size - 08 DASH.

Table 2 shows difference in fluid pressure in the outlet and inlet at different fitting connections. Experimental data on each standard of fitting are presented. Three instances have been provided to eliminate data distortion.

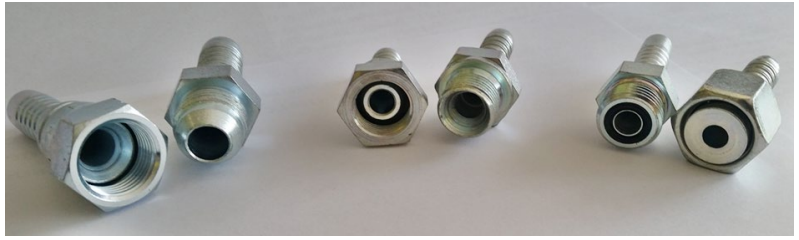

Figure 2. View of the standards of fitting connections

a)

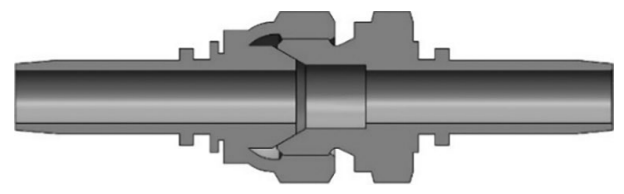

b)

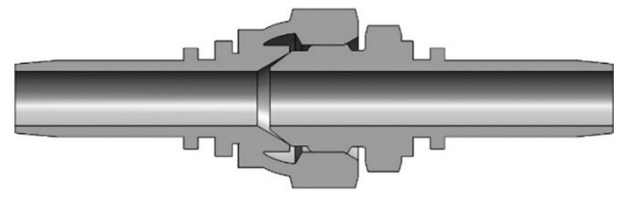

c)

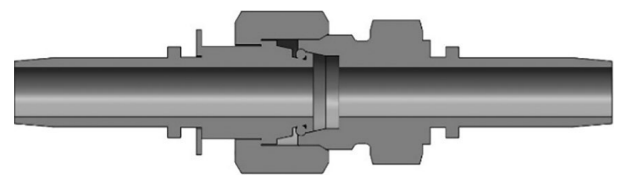

d)

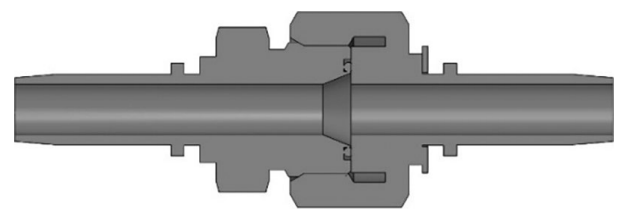

Figure 3. Cross-section standards of 3D model fitting connections: $\mathrm{a}$ - BSP; $\mathrm{b}$ - JIS; $\mathrm{c}$ - DKOL; $\mathrm{d}$ - ORFS

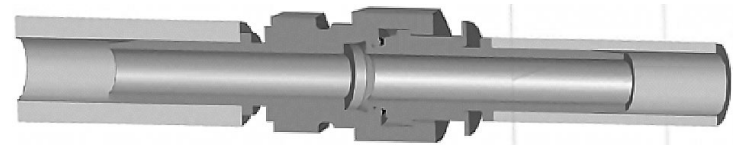

Figure 4. Cross-section cut in the HPH and DKOL fitting connection
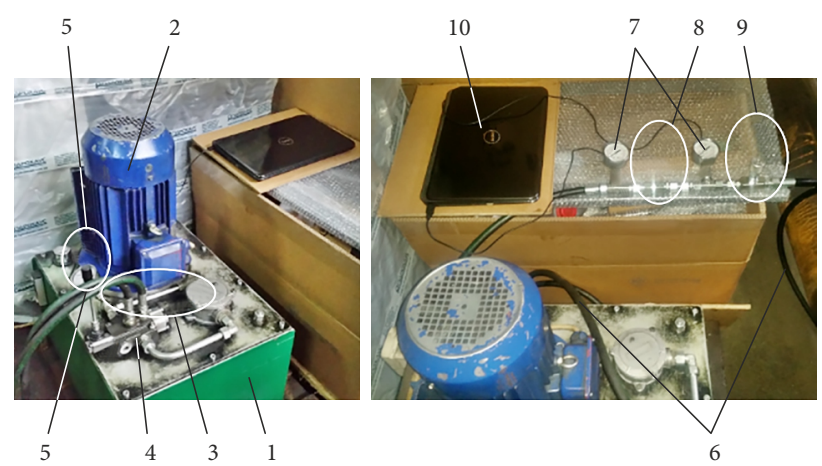

Figure 5. The experimental bench for investigating pressure losses at fitting connections

Experimental data on observation have disclosed highest pressure losses at BSP fitting connections $(\sim 4870 \mathrm{~Pa})$, DKOL comes next ( $\sim 540 \mathrm{~Pa}$ ), ORFS makes $\sim 4180 \mathrm{~Pa}$ and the lowest pressure losses have been found at JIS connections $(\sim 3880 \mathrm{~Pa})$. 
Table 2. Pressure drop at different fitting connections with reference to pre-experimental research

\begin{tabular}{|c|c|}
\hline Fitting standard & Pressure drop [Pa] \\
\hline \multirow{3}{*}{ BSP } & 4860 \\
\cline { 2 - 2 } & 4890 \\
\cline { 2 - 2 } & 4870 \\
\hline \multirow{3}{*}{ JIS } & 3870 \\
\cline { 2 - 2 } & 3890 \\
\hline \multirow{4}{*}{ DKOL } & 3880 \\
\cline { 2 - 2 } & 4500 \\
\cline { 2 - 2 } & 4580 \\
\hline \multirow{3}{*}{ ORFS } & 4550 \\
\hline \multirow{2}{*}{} & 4190 \\
\cline { 2 - 2 } & 4180 \\
\hline
\end{tabular}

\section{Fitting connections simulated applying CFD}

\subsection{Movement and continuity equations}

Fluid movement is considered to be 3D. All local velocity is equal to average velocity and remains unsettled. Velocity and pressure depend on coordinates and time. The dynamics of the compressible and Newtonian fluid flow is governed by Navier-Stokes equations and represented by the conservation of momentum. Thus, from mass conservation, the divergence of the velocity field is equal to zero $(\nabla u=0)$ (Rodrigues Santos et al. 2018). Movement and continuity equations for a viscous, compressible fluid in the pressure pipe have the following form (Foias et al. 2001):

$$
\begin{aligned}
& \frac{\partial(\rho \cdot u)}{\partial t}+\frac{\partial\left(\rho \cdot u^{2}\right)}{\partial x}+\frac{\partial(\rho \cdot u \cdot v)}{\partial y}+\frac{\partial(\rho \cdot u \cdot w)}{\partial z}= \\
& -\frac{\partial p}{\partial x}+\frac{1}{R e} \cdot\left(\frac{\partial \tau_{x x}}{\partial x}+\frac{\partial \tau_{x y}}{\partial y}+\frac{\partial \tau_{x z}}{\partial z}\right) ; \\
& \frac{\partial(\rho \cdot v)}{\partial t}+\frac{\partial(\rho \cdot u \cdot v)}{\partial x}+\frac{\partial\left(\rho \cdot v^{2}\right)}{\partial y}+\frac{\partial(\rho \cdot v \cdot w)}{\partial z}= \\
& -\frac{\partial p}{\partial y}+\frac{1}{R e} \cdot\left(\frac{\partial \tau_{x y}}{\partial x}+\frac{\partial \tau_{y y}}{\partial y}+\frac{\partial \tau_{y z}}{\partial z}\right) ; \\
& \frac{\partial(\rho \cdot w)}{\partial t}+\frac{\partial(\rho \cdot u \cdot w)}{\partial x}+\frac{\partial(\rho \cdot v \cdot w)}{\partial y}+\frac{\partial\left(\rho \cdot w^{2}\right)}{\partial z}= \\
& -\frac{\partial p}{\partial z}+\frac{1}{R e} \cdot\left(\frac{\partial \tau_{x z}}{\partial x}+\frac{\partial \tau_{y z}}{\partial y}+\frac{\partial \tau_{z z}}{\partial z}\right) ; \\
& \frac{\partial \rho}{\partial t}+\frac{\partial(\rho \cdot u)}{\partial x}+\frac{\partial(\rho \cdot v)}{\partial y}+\frac{\partial(\rho \cdot w)}{\partial z}=0 .
\end{aligned}
$$

Computations were carried out employing commercial CFD software Ansys ${ }^{\circledR}$ Fluent $^{\circledR}$ (https://www.ansys. $\mathrm{com} /$ products/fluids/ansys-fluent). Simulation software was configured for a study of the steady-state fluid flow in
$3 \mathrm{D}$ geometry, and the standard $k-\varepsilon$ turbulence model was selected to analyse fluid flow. For the application of the standard $k-\varepsilon$ turbulence model, the following transport equations for turbulent kinetic energy $k$ and turbulent dissipation $\varepsilon$ are implemented (Launder, Spalding 1972):

$$
\begin{aligned}
& \frac{\partial}{\partial t}(\rho \cdot k)+\frac{\partial}{\partial x_{i}}\left(\rho \cdot k \cdot u_{i}\right)=\frac{\partial}{\partial x_{j}}\left(\left(\mu+\frac{\mu_{t}}{\sigma_{k}}\right) \frac{\partial k}{\partial x_{j}}\right)+ \\
& 2 \cdot \mu_{t} \cdot E_{i j} \cdot E_{i j}-\rho \cdot \varepsilon ; \\
& \frac{\partial}{\partial t}(\rho \cdot \varepsilon)+\frac{\partial}{\partial x_{i}}\left(\rho \cdot \varepsilon \cdot u_{i}\right)=\frac{\partial}{\partial x_{j}}\left(\left(\mu+\frac{\mu_{t}}{\sigma_{\varepsilon}}\right) \frac{\partial \varepsilon}{\partial x_{j}}\right)+ \\
& C_{1 \varepsilon} \cdot \frac{\varepsilon}{k} \cdot\left(2 \cdot \mu_{t} \cdot E_{i j} \cdot E_{i j}\right)-C_{2 \varepsilon} \cdot \rho \cdot \frac{\varepsilon^{2}}{k} ; \\
& \mu_{t}=\frac{\rho \cdot C_{\mu} \cdot k^{2}}{\varepsilon},
\end{aligned}
$$

where: $C_{1 \varepsilon}, C_{2 \varepsilon}, C_{\mu}$ - constants of the $k-\varepsilon$ turbulence model shown in Table 3.

The above default values have been determined from experiments on fundamental turbulent flows, including frequently encountered shear flows like boundary layers, mixing layers and jets as well as for decaying isotropic grid turbulence according to the ANSYS Fluent Theory Guide (ANSYS 2013). They found to work fairly well for a wide range of wall-bounded and free shear flows.

Table 3. The constants of the $k-\varepsilon$ turbulence model

\begin{tabular}{|c|c|c|c|c|}
\hline$C_{\mu}$ & $C_{1 \varepsilon}$ & $C_{2 \varepsilon}$ & $\sigma_{k}$ & $\mathrm{~s}_{\mathrm{e}}$ \\
\hline 0.09 & 1.44 & 1.92 & 1.00 & 1.30 \\
\hline
\end{tabular}

\subsection{The simulation model and fluid parameters}

The study used a mobile hydraulic power unit consisting of a gear pump with the fixed displacement driven by an electric motor at a constant speed in all measurements. In this case, an assumption that mass flow rate at the input of the HPH-fittings system is practically stable can be made.

Multiphase simulation involves a homogenous material, i.e. standard mineral hydraulic oil Hydraux HLP 46 that conforms to the DIN 51524-2:2016, see research by Tič and Lovrec (2012). The properties of oil are shown in Table 4.

Table 4. The properties of Hydraux HLP 46 oil

\begin{tabular}{|l|c|}
\hline \multicolumn{1}{|c|}{ Properties } & Value \\
\hline Molar mass $[\mathrm{kg} / \mathrm{kmol}]$ & 300 \\
\hline Density $\left[\mathrm{kg} / \mathrm{m}^{3}\right]$ & 874 \\
\hline Kinematic viscosity $\left[\mathrm{mm}^{2} / \mathrm{s}\right]$ & 46 \\
\hline Specific heat capacity $[\mathrm{J} / \mathrm{kg} \cdot \mathrm{K}]$ & 1966 \\
\hline Reference temperature $\left[\mathrm{C}^{\circ}\right]$ & 40 \\
\hline Reference pressure $\left[\mathrm{N} / \mathrm{mm}^{2}\right]$ & $1 \cdot 10^{5}$ \\
\hline Thermal conductivity $[\mathrm{W} / \mathrm{m} \cdot \mathrm{K}]$ & 0.292 \\
\hline
\end{tabular}




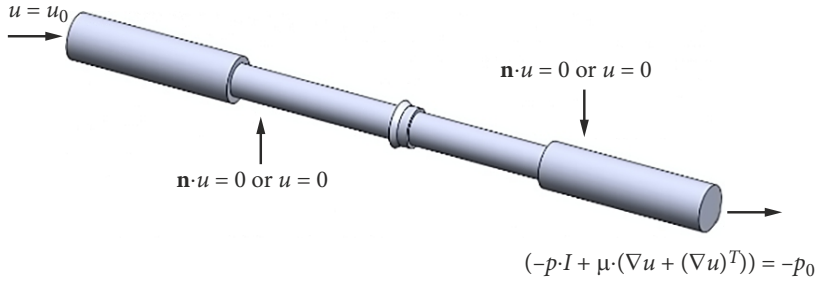

Figure 6. Boundary conditions of the pipeline-fittings system for Ansys ${ }^{\circledR}$ Fluent $^{\circledR}$ simulation

Boundary conditions. Several boundary conditions are used for solving the compressible Navier-Stokes equations where $k-\varepsilon$ - turbulence model (Karpenko, Bogdevičius 2020). Figure 6 shows an example of the applied boundary conditions. Information about boundary layers covers a wall boundary condition, an inlet boundary condition and an outlet boundary condition.

Inlet boundary condition. For the inlet, a velocity vector normal to the boundary is specified as:

$$
u \cdot \mathbf{n}=u_{0}
$$

where: $\mathbf{n}$ is a unit vector having a direction perpendicular or normal to the boundary.

Outlet boundary condition. For the outlet, certain pressure in the outlet/pressure boundary condition is imposed:

$$
\begin{aligned}
& p=p_{0} \\
& \left(-p \cdot I+\mu \cdot\left(\nabla u+(\nabla u)^{T}\right)\right)=-p_{0} .
\end{aligned}
$$

Wall boundary condition. The wall boundary condition states there are no velocity components perpendicular to the boundary (due to Newtonian fluid, the velocity of fluid near the wall is equal to zero):

$$
\mathbf{n} \cdot u=0 \text { or } u=0 .
$$

\subsection{Mesh generation}

The numerical simulation of fluid flow inside fitting connections was done employing the Ansys ${ }^{\circledR}$ Workbench $^{\circledR}$. The numerical code was based on the FVM. The investigation area covered a $3 \mathrm{D}$ volume closed from all sides and divided into tetrahedrons. The dependent variables of pressure, velocity and turbulent kinetic energy as well as the volume fraction were calculated for each node of the flow-element (Biluš et al. 2005). The mesh refined near changes in the cross-section area and around restrictive objects, as necessary according to Lisowski and Panek (2004), in order to obtain more accurate experimental measurements and models shown in Figure 7a. Close to the walls, boundary layers maximally affect velocity gradients in the normal direction to the wall. Thus, ten inflation layers were created with an expansion factor of 1.2...1.6 depending on changes in diameter (Figure $7 \mathrm{~b}$ ). a)

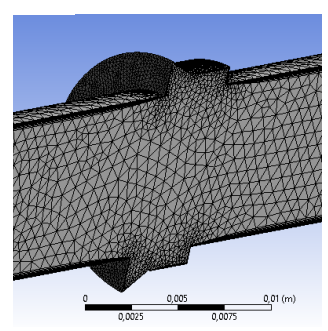

b)

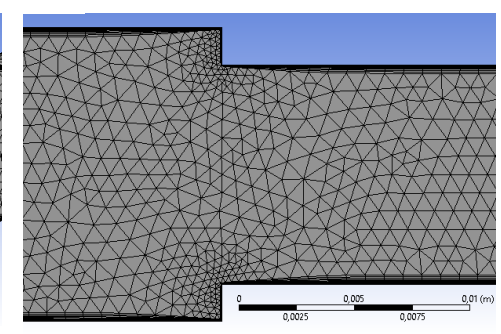

Figure 7. The mesh of the model: a - mesh generation; $\mathrm{b}$ - inflating layers with the expansion factor of $1.2 \ldots 1.6$

\subsection{Mesh independence study}

To establish the accuracy of simulating CFD and to keep computational costs low, fitting connections were analysed using the standard $k-\varepsilon$ model at uniform $V_{\text {in }}=3.12 \mathrm{~m} / \mathrm{s}$ and $p=2 \mathrm{MPa}$, which corresponded to experimental settings. The study of grid convergence was performed by developing different types of mesh for every fitting connection to determine how mesh quality affected CFD simulation results.

The number of nodes and simulation time using the standard $k-\varepsilon$ model given in Table 5 summarised the main characteristics of the mesh thus clarifying that CFD simulation time was highly dependent on the number of mesh nodes. The example provided in Figure 8 shows mesh generation for each mesh resolution on ORFS standard connection.

It is important to note that mesh resolution plays a pivotal role in the final CFD results. M5 is the closest to the experimental measurement of pressure drop obtained from the independent mesh study. M3 and M5 account for nearly $1 \%$ difference in the estimated pressure drop, but the final CFD simulation time required for convergence of two meshes has a significant difference.

From the final CFD simulation results, simulation time is particularly subject to the number of mesh nodes. Due to a slight difference between M5 and M3 is best regarding computational costs and is further employed M3 for the numerical analysis carried out in the following researching.

\section{The results of simulating ANSYS CFD}

Ansys ${ }^{\circledR}$ Fluent $^{\circledR}$ simulation shows that pressure drop at different fitting connections (Table 6) of fluid flow are taken at a rate from 5 to $100 \mathrm{~L} / \mathrm{min}$. For a better understanding and explanation of the obtained simulation results, the Re number was calculated (Figure 9). The total pressure profile of fluid on BSP/ORFS/DKOL/JIS fitting connections and the straight equivalent length of the pipeline are displayed in Figure 10. The additional results of fluent simulation on the DKOL fitting connection are provided in Figures 11 and 12. 
a)

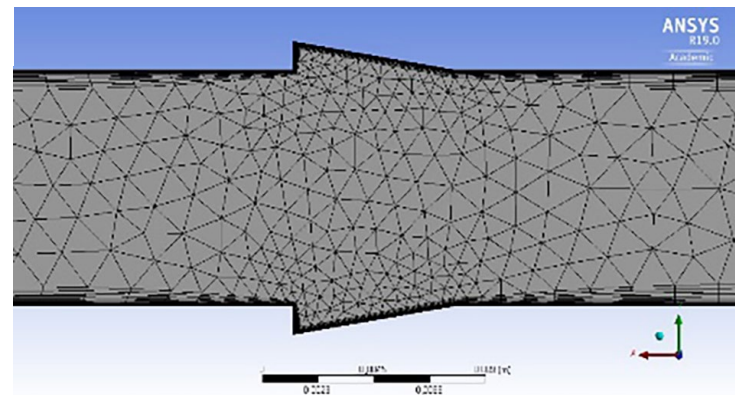

b)

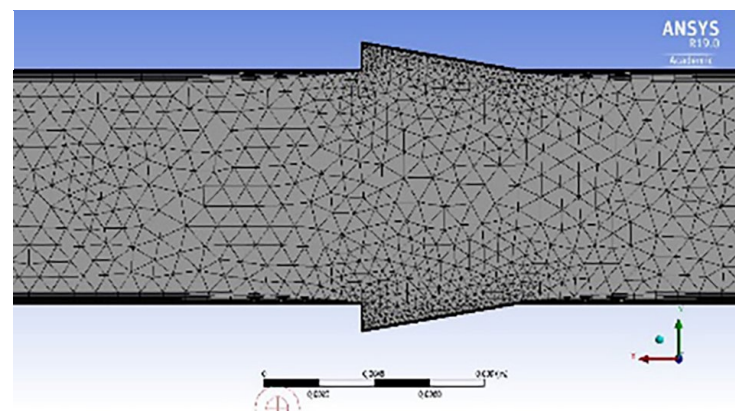

c)

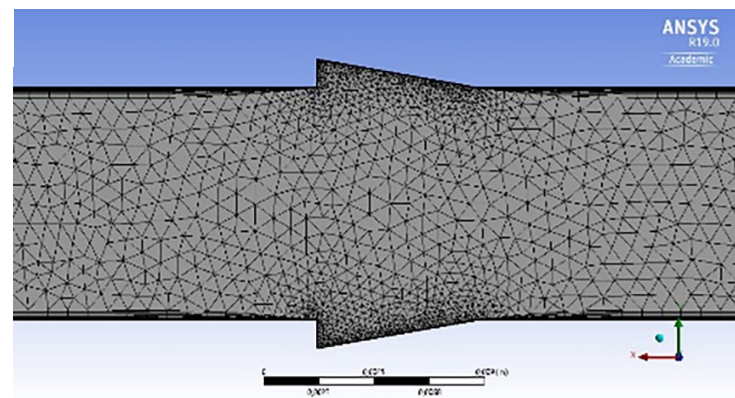

Figure 8 . Mesh resolution of the mesh independence study: a - M1; b - M3; c - M5
As noticed from the Re number provided in the chart (Figure 9), the turbulence of fluid flow through fitting connections started at the flow rate of approximately $35 \mathrm{~L} / \mathrm{min}$, and that of the pipeline of the equivalent length - at a rate of $65 \mathrm{~L} / \mathrm{min}$, which confirms that the equivalent length method is hardly accepted as an accurate technique for calculating losses on fitting connections.

All above introduced results were taken from Ansys ${ }^{\circledR}$ Fluent $^{\circledR}$ simulation wherein inlet upload velocity was $5.291 \mathrm{~m} / \mathrm{s}$, which corresponded to the flow rate of $40 \mathrm{~L} / \mathrm{min}$. The obtained results are presented to show fluid flow turbulence processes inside fitting connections.

The resistance coefficient of fitting connections was calculated from pressure losses (the results of calculating resistance coefficients are shown in Table 7):

$$
\xi=\frac{2 \cdot \Delta p}{\rho \cdot v_{\text {average }}^{2}} .
$$

Calculation was done separately for each standard of connection at a different flow rate.

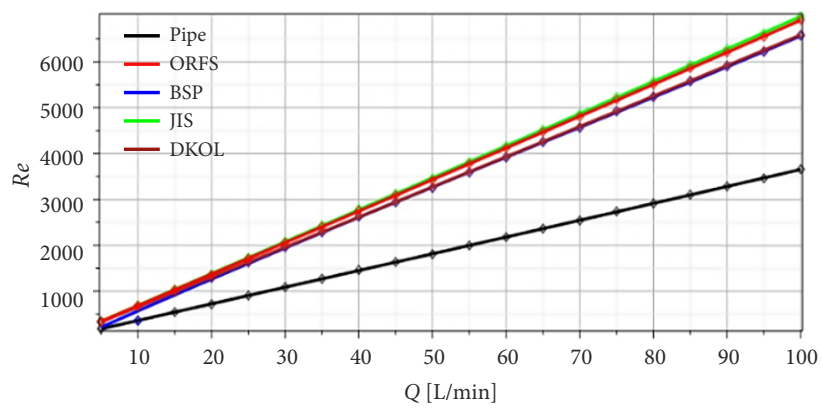

Figure 9. The chart of in depending Re number from flow rate for different fitting connections

Table 5. The results of the mesh independency study

\begin{tabular}{|c|c|c|c|c|c|c|}
\hline \multirow{2}{*}{$\begin{array}{l}\text { Number of } \\
\text { the mesh }\end{array}$} & \multirow{2}{*}{$\begin{array}{l}\text { Mesh } \\
\text { resolution }\end{array}$} & \multirow{2}{*}{$\begin{array}{l}\text { Straight } \\
\text { pipeline }\end{array}$} & \multicolumn{4}{|c|}{ Fitting connection } \\
\hline & & & BSP & JIS & DKOL & ORFS \\
\hline \multirow{3}{*}{ M1 } & number of elements & 112896 & 189628 & 192587 & 187568 & 191227 \\
\hline & CFD simulation time [h:min] & $0: 16$ & $0: 32$ & $0: 28$ & $0: 22$ & $0: 22$ \\
\hline & estimated $\Delta p[\mathrm{~Pa}]$ & 2320 & 3840 & 3290 & 4190 & 3810 \\
\hline \multirow{3}{*}{ M2 } & number of elements & 157856 & 264587 & 241368 & 212478 & 210587 \\
\hline & CFD simulation time [h:min] & $0: 22$ & $0: 37$ & $0: 32$ & $0: 28$ & $0: 27$ \\
\hline & estimated $\Delta p[\mathrm{~Pa}]$ & 2890 & 3900 & 3350 & 4270 & 3970 \\
\hline \multirow{3}{*}{ M3 } & number of elements & 289536 & 489632 & 449632 & 421236 & 419587 \\
\hline & CFD simulation time [h:min] & $0: 32$ & $0: 46$ & $0: 39$ & $0: 36$ & $0: 34$ \\
\hline & estimated $\Delta p[\mathrm{~Pa}]$ & 3690 & 4490 & 3860 & 4360 & 4160 \\
\hline \multirow{3}{*}{ M4 } & number of elements & 369852 & 544127 & 537885 & 522563 & 520865 \\
\hline & CFD simulation time [h:min] & $0: 43$ & $1: 12$ & $0: 51$ & $0: 47$ & $0: 46$ \\
\hline & estimated $\Delta p[\mathrm{~Pa}]$ & 3700 & 4500 & 3860 & 4390 & 4160 \\
\hline \multirow{3}{*}{ M5 } & number of elements & 508698 & 785696 & 747896 & 731583 & 730112 \\
\hline & CFD simulation time [h:min] & $0: 58$ & $1: 44$ & $1: 28$ & $1: 17$ & $1: 08$ \\
\hline & estimated $\Delta p[\mathrm{~Pa}]$ & 3710 & 4520 & 3870 & 4400 & 4170 \\
\hline
\end{tabular}


Table 6. The results of fluent simulation (pressure drop)

\begin{tabular}{|c|c|c|c|c|c|c|}
\hline \multirow{2}{*}{$\begin{array}{l}\text { Flow rate } \\
Q[\mathrm{~L} / \mathrm{min}]\end{array}$} & \multirow{2}{*}{$\begin{array}{c}\text { Average velocity of fluid flow } \\
v_{\text {average }}[\mathrm{m} / \mathrm{s}]\end{array}$} & \multicolumn{5}{|c|}{ Pressure drop $\Delta p[\mathrm{~Pa}]$} \\
\hline & & Pipe & BSP & JIS & DKOL & ORFS \\
\hline 5 & 0.657 & 198 & 243 & 208 & 233 & 211 \\
\hline 10 & 1.318 & 795 & 970 & 837 & 939 & 842 \\
\hline 15 & 1.974 & 1777 & 2166 & 1871 & 2097 & 1878 \\
\hline 20 & 2.6 & 3079 & 3748 & 3221 & 3633 & 3234 \\
\hline 25 & 3.28 & 4898 & 5396 & 5084 & 5105 & 5093 \\
\hline 30 & 3.95 & 7096 & 7520 & 7335 & 7356 & 7348 \\
\hline 35 & 4.61 & 9661 & 10127 & 9894 & 9945 & 9911 \\
\hline 40 & 5.291 & 12713 & 13207 & 12982 & 13033 & 12996 \\
\hline 45 & 5.923 & 15888 & 16489 & 16232 & 16287 & 16268 \\
\hline 50 & 6.55 & 19358 & 20088 & 19839 & 19906 & 19873 \\
\hline 55 & 7.234 & 23587 & 24447 & 24186 & 24267 & 24226 \\
\hline 60 & 7.89 & 28029 & 29065 & 28755 & 28860 & 28804 \\
\hline 65 & 8.57 & 32981 & 34233 & 33925 & 34040 & 33944 \\
\hline 70 & 9.2 & 37948 & 39384 & 39075 & 39206 & 39097 \\
\hline 75 & 9.92 & 44074 & 45764 & 45380 & 45558 & 45430 \\
\hline 80 & 10.55 & 49719 & 51615 & 50709 & 51441 & 51370 \\
\hline 85 & 11.238 & 56298 & 58534 & 57508 & 58337 & 58272 \\
\hline 90 & 11.9 & 63061 & 65596 & 64448 & 65376 & 65303 \\
\hline 95 & 12.54 & 69990 & 72801 & 71219 & 72436 & 72476 \\
\hline 100 & 13.25 & 78140 & 81232 & 79470 & 80647 & 80870 \\
\hline
\end{tabular}

a)

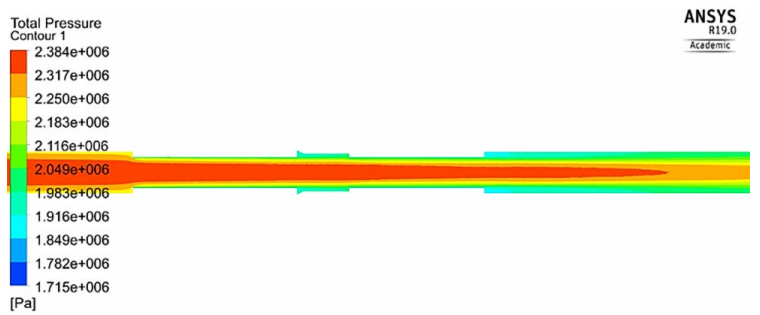

b)

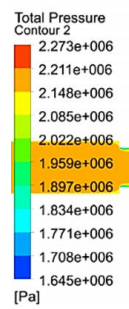

d)

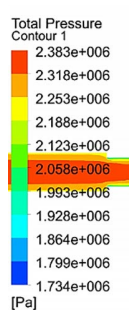

c)

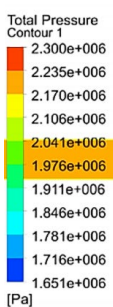

$+006$

$1.651++006$

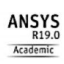
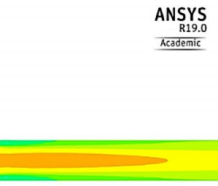

$$
006
$$

e)

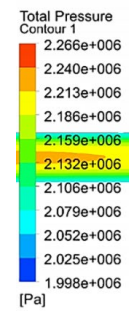

ANSYS

Figure 10. Total fluid pressure across fitting connections (by fluent simulation): a - BSP fitting connection; b - JIS fitting connection; $c$ - DKOL fitting connection; $d$ - ORFS fitting connection; $\mathrm{e}$ - straight pipeline of the equivalent length 
Table 7. The results of fluent simulation (resistance coefficient)

\begin{tabular}{|c|c|c|c|c|c|c|}
\hline \multirow{2}{*}{$\begin{array}{l}\text { Flow rate } \\
Q[\mathrm{~L} / \mathrm{min}]\end{array}$} & \multirow{2}{*}{$\begin{array}{l}\text { Average velocity of } \\
\text { fluid flow } v_{\text {average }}[\mathrm{m} / \mathrm{s}]\end{array}$} & \multicolumn{5}{|c|}{ Resistance coefficient $\xi$} \\
\hline & & Pipe & BSP & JIS & DKOL & ORFS \\
\hline 5 & 0.657 & 1.054 & 1.288 & 1.103 & 1.238 & 1.119 \\
\hline 10 & 1.318 & 1.048 & 1.278 & 1.102 & 1.237 & 1.109 \\
\hline 15 & 1.974 & 1.043 & 1.272 & 1.098 & 1.231 & 1.102 \\
\hline 20 & 2.6 & 1.042 & 1.268 & 1.090 & 1.229 & 1.095 \\
\hline 25 & 3.28 & 1.041 & 1.147 & 1.081 & 1.085 & 1.083 \\
\hline 30 & 3.95 & 1.0408 & 1.102 & 1.075 & 1.078 & 1.077 \\
\hline 35 & 4.61 & 1.0403 & 1.091 & 1.065 & 1.070 & 1.067 \\
\hline 40 & 5.291 & 1.039 & 1.079 & 1.061 & 1.065 & 1.062 \\
\hline 45 & 5.923 & 1.036 & 1.075 & 1.0588 & 1.062 & 1.061 \\
\hline 50 & 6.55 & 1.032 & 1.071 & 1.0582 & 1.0617 & 1.0599 \\
\hline 55 & 7.234 & 1.031 & 1.069 & 1.0576 & 1.061 & 1.059 \\
\hline 60 & 7.89 & 1.030 & 1.068 & 1.0572 & 1.060 & 1.058 \\
\hline 65 & 8.57 & 1.027 & 1.066 & 1.057 & 1.0605 & 1.0576 \\
\hline 70 & 9.2 & 1.025 & 1.064 & 1.056 & 1.0599 & 1.057 \\
\hline 75 & 9.92 & 1.024 & 1.064 & 1.055 & 1.059 & 1.0564 \\
\hline 80 & 10.55 & 1.022 & 1.061 & 1.0425 & 1.0576 & 1.056 \\
\hline 85 & 11.238 & 1.02 & 1.060 & 1.042 & 1.057 & 1.0558 \\
\hline 90 & 11.9 & 1.019 & 1.059 & 1.041 & 1.056 & 1.055 \\
\hline 95 & 12.54 & 1.018 & 1.059 & 1.036 & 1.0546 & 1.054 \\
\hline 100 & 13.25 & 1.018 & 1.058 & 1.035 & 1.054 & 1.051 \\
\hline
\end{tabular}

a) velocity

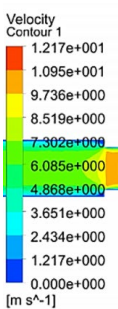

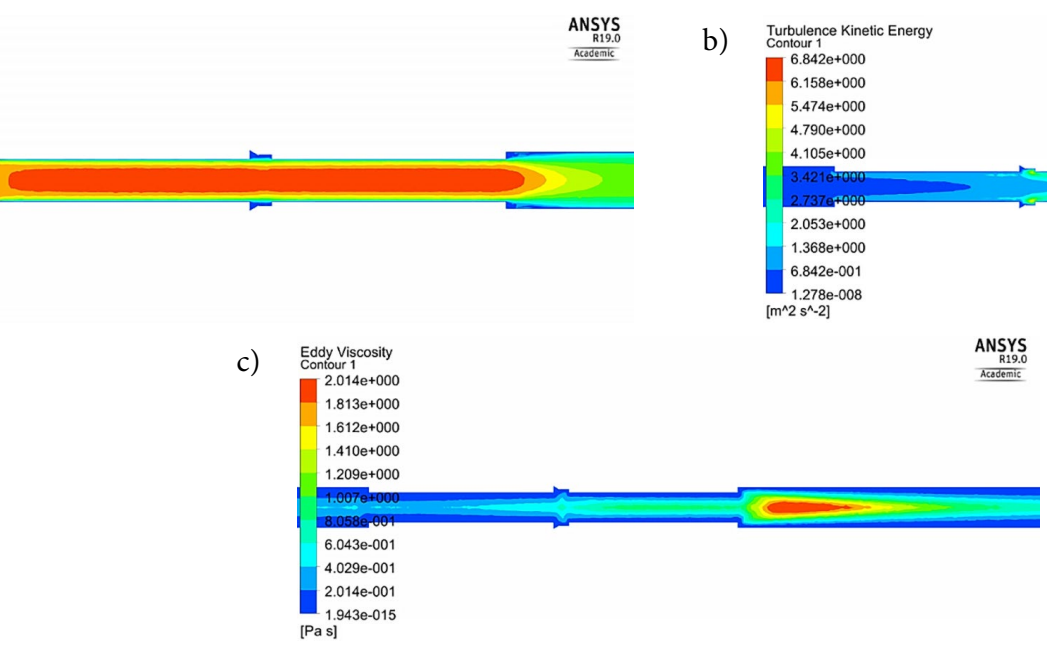

Figure 11. Additional results from the fluent simulation of the DKOL fitting connection: a - velocity;

$\mathrm{b}$ - turbulence kinetic energy; c - eddy viscosity

The flow coefficient of a device is a relative measure of its efficiency at an allowed fluid flow. The coefficient describes the relationship between pressure drop across the orifice, valve or other assembly and the corresponding flow rate:

$$
\mu=\frac{Q}{A \cdot \sqrt{\frac{2 \cdot \Delta p}{b}}},
$$

where: $b=1-\left(\frac{d}{D}\right)^{4}$.
For different fitting connections, the flow coefficient is given in Table 8 and Figure 12.

Power losses (Table 9) of each standard of the fitting connection of the hydraulic system are calculated as:

$N_{L}=\Delta p \cdot Q$.

Research objects are divided into the following groups:

- Group 1 - a straight pipeline with the equivalent length of fitting connections;

- Group 2 - JIS and ORFS standards of fitting connections; 
- Group 3 - BSP and DKOL standards of fitting connections.

The obtained results showed that Group 1 had the most optimal flow characteristics (flow coefficient ranged from 0.9 to 0.964 ). Group 2 included the JIS (flow coefficient ranged from 0.821 to 0.931 ) and the ORFS (flow coefficient ranged from 0.798 to 0.9 ) and performed worse. Flow characteristics of Group 3 embraced the DKOL (flow coefficient ranged from 0.652 to 0.9 ) and the BSP (flow coefficient ranged from 0.602 to 0.892 ) and performed the least effectively, which was due to significant changes in the cross-section area of the standards of fitting connections. Group 1 presented an ideal section of the pipeline with no changes in the cross-section area, whereas Groups 2 and 3 pointed out changes in cross-section areas.

The difference between changes in the cross-section areas of Groups 2 and 3 had a significant impact on flow characteristics. Changes in the cross-section areas of Group 3 were higher than those in Group 2. Figure 12 shows difference between flow coefficients, which made around 25\% for Groups 2 and 3 at the beginning of the chart. Also, a significant difference in flow characteristics at different flow processes inside fitting connections is observed. Unlike Groups 2 and 3, Group 1 had no significant differences in the processes of the laminar or turbulent flow (according to the uploaded boundary conditions). Group 3 has the worst characteristics of the laminar flow, which is less than 25\% established in Group 2 and less than $30 \%$ determined in Group 1. However, in terms of the turbulence of flow processes, Groups 2 and 3 have same flow characteristics. The difference in the flow coefficient, because of flow turbulence, between Group 1 and Groups 2 and 3 was around 10\%. This proves that changes in the cross-section areas inside fitting connections had less influence than the turbulence of the inside flow.

Research on power losses in Group 1 demonstrated insignificant power losses (from $1.61 \cdot 10^{-3}$ to $11.041 \mathrm{~W}$ ) compared to Group 2 that included JIS (ranged from $1.73 \cdot 10^{-3}$ to $13.271 \mathrm{~W}$ ) and ORFS (ranged from $1.758 \cdot 10^{-3}$ to $13.505 \mathrm{~W})$. Higher power losses were observed in Group 3 that embraced DKOL (ranged from $1.94 \cdot 10^{-3}$ to $13.468 \mathrm{~W}$ ) and BSP (ranged from $2.12 \cdot 10^{-3}$ to $13.562 \mathrm{~W}$ ). Even though the difference in power losses between Group 1 and Groups 2 and 3 is approximately 50\% due to laminar flow processes, the difference in turbulence makes only $10 \ldots 20 \%$.

The introduced model shows that turbulent kinetic energy grows up to $1.27 \cdot 10^{-8} \mathrm{~m}^{2} / \mathrm{s}^{2}$ at the inlet of DKOL fitting connections and up to $6.84 \mathrm{~m}^{2} / \mathrm{s}^{2}$ at the outlet of fitting connections, which produces severe turbulence in the system at the flow rate of $40 \mathrm{~L} / \mathrm{min}$.

The conducted research disclosed that standard methods for calculating hydraulic fittings were not accurate, and each type of fitting connections required additional investigations.

Table 8 . The results of fluent simulation (flow coefficient)

\begin{tabular}{|c|c|c|c|c|c|c|}
\hline \multirow{2}{*}{$\begin{array}{l}\text { Flow rate } \\
Q[\mathrm{~L} / \mathrm{min}]\end{array}$} & \multirow{2}{*}{$\begin{array}{l}\text { Average velocity of } \\
\text { fluid flow } v_{\text {average }}[\mathrm{m} / \mathrm{s}]\end{array}$} & \multicolumn{5}{|c|}{ Flow coefficient $\mu$} \\
\hline & & Pipe & BSP & JIS & DKOL & ORFS \\
\hline 5 & 0.657 & 0.9 & 0.602 & 0.821 & 0.652 & 0.798 \\
\hline 10 & 1.318 & 0.91 & 0.612 & 0.822 & 0.653 & 0.812 \\
\hline 15 & 1.974 & 0.918 & 0.618 & 0.828 & 0.659 & 0.822 \\
\hline 20 & 2.6 & 0.92 & 0.621 & 0.841 & 0.661 & 0.834 \\
\hline 25 & 3.28 & 0.921 & 0.759 & 0.855 & 0.848 & 0.852 \\
\hline 30 & 3.95 & 0.923 & 0.822 & 0.864 & 0.859 & 0.861 \\
\hline 35 & 4.61 & 0.924 & 0.841 & 0.881 & 0.872 & 0.878 \\
\hline 40 & 5.291 & 0.926 & 0.858 & 0.888 & 0.881 & 0.886 \\
\hline 45 & 5.923 & 0.931 & 0.865 & 0.892 & 0.886 & 0.888 \\
\hline 50 & 6.55 & 0.938 & 0.871 & 0.893 & 0.887 & 0.890 \\
\hline 55 & 7.234 & 0.94 & 0.875 & 0.894 & 0.888 & 0.891 \\
\hline 60 & 7.89 & 0.942 & 0.876 & 0.895 & 0.888 & 0.892 \\
\hline 65 & 8.57 & 0.947 & 0.879 & 0.895 & 0.889 & 0.894 \\
\hline 70 & 9.2 & 0.95 & 0.882 & 0.896 & 0.89 & 0.895 \\
\hline 75 & 9.92 & 0.952 & 0.883 & 0.898 & 0.891 & 0.896 \\
\hline 80 & 10.55 & 0.957 & 0.888 & 0.920 & 0.894 & 0.896 \\
\hline 85 & 11.238 & 0.961 & 0.889 & 0.921 & 0.895 & 0.897 \\
\hline 90 & 11.9 & 0.963 & 0.890 & 0.922 & 0.896 & 0.898 \\
\hline 95 & 12.54 & 0.964 & 0.891 & 0.931 & 0.9 & 0.899 \\
\hline 100 & 13.25 & 0.964 & 0.892 & 0.931 & 0.9 & 0.9 \\
\hline
\end{tabular}


Table 9. Power losses of each standard of fitting connections

\begin{tabular}{|c|c|c|c|c|c|c|}
\hline \multirow{2}{*}{$\begin{array}{l}\text { Flow rate } \\
Q[\mathrm{~L} / \mathrm{min}]\end{array}$} & \multirow{2}{*}{$\begin{array}{c}\text { Average velocity of fluid flow } \\
v_{\text {average }}[\mathrm{m} / \mathrm{s}]\end{array}$} & \multicolumn{5}{|c|}{ Power losses $N_{L}[\mathrm{~W}]$} \\
\hline & & Pipe & BSP & JIS & DKOL & ORFS \\
\hline 5 & 0.657 & $1.61 \cdot 10^{-3}$ & $2.12 \cdot 10^{-3}$ & $1.73 \cdot 10^{-3}$ & $1.94 \cdot 10^{-3}$ & $1.758 \cdot 10^{-3}$ \\
\hline 10 & 1.318 & $1.32 \cdot 10^{-2}$ & $1.74 \cdot 10^{-2}$ & $1.39 \cdot 10^{-2}$ & $1.56 \cdot 10^{-2}$ & $1.41 \cdot 10^{-2}$ \\
\hline 15 & 1.974 & $4.31 \cdot 10^{-2}$ & $5.41 \cdot 10^{-2}$ & $4.67 \cdot 10^{-2}$ & $5.24 \cdot 10^{-2}$ & $4.69 \cdot 10^{-2}$ \\
\hline 20 & 2.6 & 0.112 & 0.134 & 0.106 & 0.119 & 0.1067 \\
\hline 25 & 3.28 & 0.213 & 0.232 & 0.211 & 0.212 & 0.211 \\
\hline 30 & 3.95 & 0.351 & 0.381 & 0.366 & 0.367 & 0.367 \\
\hline 35 & 4.61 & 0.562 & 0.605 & 0.576 & 0.579 & 0.577 \\
\hline 40 & 5.291 & 0.454 & 0.892 & 0.869 & 0.873 & 0.870 \\
\hline 45 & 5.923 & 1.0197 & 1.248 & 1.217 & 1.221 & 1.220 \\
\hline 50 & 6.55 & 1.0622 & 1.677 & 1.646 & 1.652 & 1.649 \\
\hline 55 & 7.234 & 1.565 & 2.241 & 2.215 & 2.222 & 2.219 \\
\hline 60 & 7.89 & 2.111 & 2.912 & 2.875 & 2.886 & 2.880 \\
\hline 65 & 8.57 & 2.563 & 3.696 & 3.663 & 3.676 & 3.666 \\
\hline 70 & 9.2 & 3.402 & 4.565 & 4.532 & 4.547 & 4.535 \\
\hline 75 & 9.92 & 4.511 & 5.723 & 5.672 & 5.694 & 5.678 \\
\hline 80 & 10.55 & 5.609 & 6.871 & 6.744 & 6.841 & 6.832 \\
\hline 85 & 11.238 & 7.967 & 8.297 & 8.143 & 8.260 & 8.251 \\
\hline 90 & 11.9 & 8.462 & 9.845 & 9.667 & 9.806 & 9.795 \\
\hline 95 & 12.54 & 10.052 & 11.511 & 11.252 & 11.444 & 11.451 \\
\hline 100 & 13.25 & 11.041 & 13.562 & 13.271 & 13.468 & 13.505 \\
\hline
\end{tabular}

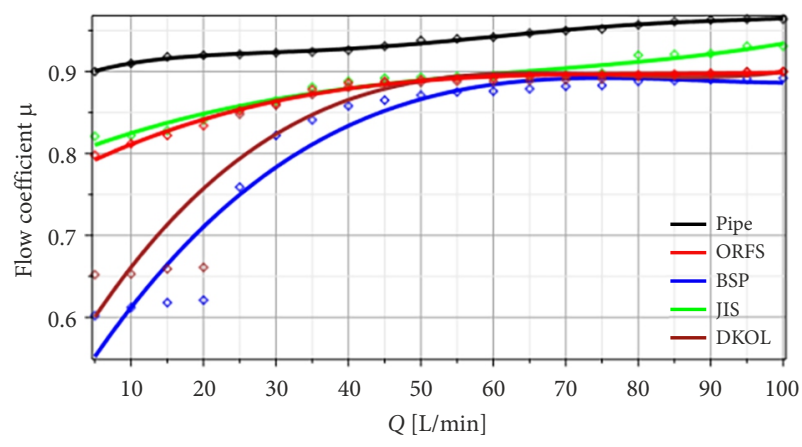

Figure 12. A chart of the flow coefficient at a different flow rate of fitting connections

\section{Conclusions}

With reference to theoretical research on simulating the hydrodynamic processes of different standards of fitting connections and straight pipeline, the obtained results showed that due to changes in the size and configuration of the cross-section area, flow velocity, the separation of transit flow from the walls of the channel and the formation of vortex occur at fitting connections. As a result, pressure drop at different fluid flow rates (from 5 to $100 \mathrm{~L} / \mathrm{min}$ ), and hence resistance and flow coefficients are determined. The mesh independence study provides that simulation time is highly dependent on the number of mesh nodes and the difference between M3 and M5 is nearly $1 \%$. However, the final CFD simulation time required for the convergence of two meshes has a significant difference.
The research objects were divided into 3 groups: Group 1 included the straight pipeline with the equivalent length of fitting connections, Group 2 embraced JIS and ORFS and Group 3 covered BSP and DKOL standards of fitting connections. The carried out research demonstrated that Group 1 performed in the most efficient way (flow coefficient ranged from 0.9 to 0.964 ) and was followed by Group 2 that included the JIS (flow coefficient ranged from 0.821 to 0.931 ) and the ORFS (flow coefficient ranged from 0.798 to 0.9 ) as well as by Group 3 incorporating the DKOL (flow coefficient ranged from 0.652 to 0.9 ) and the BSP (flow coefficient ranged from 0.602 to 0.892 ). This is due to significant changes in the cross-section area of the standards of fitting connections. Group 1 presented the ideal section of the pipeline with no changes in the cross-section area. However, Groups 2 and 3 have changes in the cross-section areas. According to the research on power losses in the pipeline of the equivalent length, fewer power losses (from $1.61 \cdot 10^{-3}$ to $11.041 \mathrm{~W}$ ) compared to the JIS (from $1.73 \cdot 10^{-3}$ to $13.271 \mathrm{~W}$ ) or the ORFS (from $1.758 \cdot 10^{-3}$ to $13.505 \mathrm{~W}$ ) were observed. Higher values of power losses were calculated in the DKOL (from 1.94.10 ${ }^{-3}$ to $13.468 \mathrm{~W}$ ) and BSP (from $2.12 \cdot 10^{-3}$ to $13.562 \mathrm{~W}$ ).

Although pressure losses of a single fitting connection are not large, modern transport vehicles contain over 200 different fitting connections that significantly affect the resistance and losses of the hydraulic system. The difference between the data obtained during the simulation of the DKOL (pressure drop of $4360 \mathrm{~Pa}$ at M3 in the mesh independence study) and pre-experimental data (pressure 
drop of around $4550 \mathrm{~Pa}$ at the fluid flow of $24 \mathrm{~L} / \mathrm{min}$ ) makes around $5 \%$.

The research identified that turbulence started in fitting connections at a range of $35 \mathrm{~L} / \mathrm{min}$, which explained a significant jump in the flow coefficient. As for the straight pipeline of the equivalent length, turbulent processes started following $65 \mathrm{~L} / \mathrm{min}$, because changes in the hydraulic diameter hardly occurred in the case of the straight pipeline of the equivalent length.

The performed research approved that using the equivalent length method is incorrect for investigation into fitting connections. Thus, for the future research, flow characteristics of the hydraulic connection on backflow would be worth investigating, because fluid moves in both directions of connections.

\section{References}

Akin, A.; Kahveci, H. S. 2019. Effect of turbulence modeling for the prediction of flow and heat transfer in rotorcraft avionics bay, Aerospace Science and Technology, 95: 105453. https://doi.org/10.1016/j.ast.2019.105453

ANSYS. 2013. ANSYS Fluent Theory Guide. Release 15.0. ANSYS, Inc. Canonsburg, PA, US. $814 \mathrm{p}$.

Biluš, I.; Škerget, L.; Predin, A.; Hriberšek, M. 2005. Experimental and numerical analyses of the cavitational flows around a hydrofoil, Strojniški vestnik - Journal of Mechanical Engineering 51(2): 103-118.

Bojko, M.; Kozubková, M. 2018. Investigation of hydraulic fitting losses, MATEC Web of Conferences 168: 02011. https://doi.org/10.1051/matecconf/201816802011

BS EN 10226-2:2005. Pipe Threads Where Pressure Tight Joints are Made on the Threads - Taper External Threads and Taper Internal Threads - Dimensions, Tolerances and Designation. British Standards Institution.

Catellani, C.; Cazzoli, G.; Falfari, S.; Forte, C.; Bianchi, G. M. 2016. Large eddy simulation of a steady flow test bench using OpenFOAM $^{\circledR}$, Energy Procedia 101: 622-629. https://doi.org/10.1016/j.egypro.2016.11.079

Crane Co. 1982. Flow of Fluids: Through Valves, Fittings, and Pipe. Metric Edition. Technical Paper No 410 M. Crane Co, New York, $133 \mathrm{p}$.

De Moraes, M. S.; Torneiros, D. L. M.; Da Silva Rosa, V.; Higa, J. S.; De Castro, Y. R.; Santos, A. R.; De Almeida Coelho, N. M.; De Moraes Júnior, D. 2017. Experimental quantification of the head loss coefficient $K$ for fittings and semi-industrial pipe cross section solid concentration profile in pneumatic conveying of polypropylene pellets in dilute phase, Powder Technology 310: 250-263.

https://doi.org/10.1016/j.powtec.2017.01.039

DIN 20066:2016. Hydraulic Fluid Power - Hose Assemblies - Dimensions, Requirements. German Institute for Standardisation.

DIN 51524-2:2016. Pressure Fluids - Hydraulic Oils - Part 2: HLP Hydraulic Oils, Minimum Requirements. German Institute for Standardisation.

Foias, C.; Manley, O.; Rosa, R.; Temam, R. 2001. Navier-Stokes Equations and Turbulence. Cambridge University Press. 364 p.

Gai, Y.; Kimiabeigi, M.; Chong, Y. C.; Widmer, J. D.; Deng, X.; Popescu, M.; Goss, J.; Staton, D. A.; Steven, A. 2019. Cooling of automotive traction motors: schemes, examples, and computation methods, IEEE Transactions on Industrial Electronics 66(3): 1681-1692. https://doi.org/10.1109/TIE.2018.2835397

Han, H.-Z.; Li, B.-X.; Li, F.-C.; He, Y.-R. 2014. RST model for turbulent flow and heat transfer mechanism in an outward convex corrugated tube, Computers \& Fluids 91: 107-129. https://doi.org/10.1016/j.compfluid.2013.12.012

JIS B 8363:2015. End Fittings and Adapters for Hydraulic Hose Assemblies. Japanese Standards Association.

Karpenko, M.; Bogdevičius, M. 2018. Investigation of hydrodynamic processes in the system - "axial piston pumps - pipeline - fittings", in Transport Problems 2018: VII International Symposium of Young Researchers, 25-26 June 2018, Katowice, Poland, 832-843.

Karpenko, M.; Bogdevičius, M. 2020. Investigation of hydrodynamic processes in the system - "pipeline-fittings", in K. Gopalakrishnan, O. Prentkovskis, I. Jackiva, R. Junevičius (Eds.). TRANSBALTICA XI: Transportation Science and Technology. TRANSBALTICA 2019. Lecture Notes in Intelligent Transportation and Infrastructure, 331-340.

https://doi.org/10.1007/978-3-030-38666-5_35

Khalizadeh, A.; Ge, H.; Ng, H. D. 2019. Effect of turbulence modeling schemes on wind-driven rain deposition on a mid-rise building: CFD modeling and validation, Journal of Wind Engineering and Industrial Aerodynamics 184: 362-377. https://doi.org/10.1016/j.jweia.2018.11.012

Launder, B.; Spalding, D. 1972. Lectures in Mathematical Models of Turbulence. Academic Press. 169 p.

Li, D.; Fu, X.; Zuo, Z.; Wang, H.; Li, Z.; Liu, S.; Wei, X. 2019. Investigation methods for analysis of transient phenomena concerning design and operation of hydraulic-machine systems a review, Renewable and Sustainable Energy Reviews 101: 26-46. https://doi.org/10.1016/j.rser.2018.10.023

Lisowski, E.; Panek, M. 2004. Modelowanie metodą CFD pracy łopatek w pompie łopatkowej, Eksploatacja i Niezawodność Maintenance and Reliability (2): 36-41. (in Polish).

Liu, H.; Zhang, X.; Quan, L.; Zhang, H. 2020. Research on energy consumption of injection molding machine driven by five different types of electro-hydraulic power units, Journal of Cleaner Production 242: 118355. https://doi.org/10.1016/j.jclepro.2019.118355

Nishimura, S.; Matsunaga, T. 2000. Analysis of response lag in hydraulic power steering system, JSAE Review 21(1): 41-46. https://doi.org/10.1016/S0389-4304(99)00059-4

Pechánek, R. Bouzek, L. 2012. Analyzing of two types water cooling electric motors using computational fluid dynamics, in 2012 15th International Power Electronics and Motion Control Conference (EPE/PEMC), 4-6 September 2012, Novi Sad, Serbia, LS2e.4-1-LS2e.4-5. https://doi.org/10.1109/EPEPEMC.2012.6397424

Rodrigues Santos, F.; Brito, A. A.; De Castro, A. P. N.; Almeida, M. P.; Da Cunha Lima, A. T.; Zebende, G. F.; da Cunha Lima, I. C. 2018. Detection of the persistency of the blockages symmetry influence on the multi-scale cross-correlations of the velocity fields in internal turbulent flows in pipelines, Physica A: Statistical Mechanics and its Applications 509: 294301. https://doi.org/10.1016/j.physa.2018.06.009

SAE J1453/3_201206. 2012. Specification for O-Ring Face Seal Connectors: Part 3 - Requirements, Dimensions, and Tests for Steel Unions, Bulkheads, Swivels, Braze Sleeves, Caps, and Connectors with SAE J1926-2 Inch Stud Ends. Society of Automotive Engineers (SAE) International. 
San Andres, U.; Almandoz, G.; Poza, J.; Ugalde, G. 2014. Design of cooling systems using computational fluid dynamics and analytical thermal models, IEEE Transactions on Industrial Electronics 61(8): 4383-4391.

https://doi.org/10.1109/TIE.2013.2286081

Savkiv, V.; Mykhailyshyn, R.; Maruschak, P.; Chovanec, L.; Prada, E.; Virgala, I.; Prentkovskis, O. 2019. Optimization of design parameters of Bernoulli gripper with an annular nozzle, in Transport Means 2019: Sustainability: Research and Solutions: Proceedings of the 23rd International Scientific Conference, 2-4 October 2019, Palanga, Lithuania, 423-428.

Tič, V.; Lovrec, D. 2012. Design of modern hydraulic tank using fluid flow simulation, International Journal of Simulation Modelling 11(2): 77-88. https://doi.org/10.2507/IJSIMM11(2)2.202

Valdés, J. R.; Rodríguez, J. M.; Saumell, J.; Pütz, T. 2014. A methodology for the parametric modelling of the flow coefficients and flow rate in hydraulic valves, Energy Conversion and Management 88: 598-611.

https://doi.org/10.1016/j.enconman.2014.08.057

Yan, X.; Chen, B.; Zhang, D.; Wu, C.; Luo, W. 2019. An energysaving method to reduce the installed power of hydraulic press machines, Journal of Cleaner Production 233: 538-545. https://doi.org/10.1016/j.jclepro.2019.06.084 